Paper

\title{
Delay effects on the limit cycling behavior in resonant inverters with state feedback
}

\author{
Luis Benadero $^{1 a)}$, Abdelali El Aroudi ${ }^{2}$, and Enrique Ponce ${ }^{3}$ \\ ${ }^{1}$ Departament de Física, Universitat Politècnica de Catalunya \\ C. Jordi Girona 1, 08023-Barcelona, Spain \\ ${ }^{2}$ Departament d'Enginyeria Electrònica Elèctrica i Automàtica, Universitat \\ Rovira $i$ Virgili \\ Av. Països Catalans 26, 43007-Tarragona, Spain \\ ${ }^{3}$ Instituto de Matemáticas $\&$ Dept. Matemática Aplicada II, Universidad de \\ Sevilla \\ E.T.S. Ingeniería, Camino de los Descubrimientos, 41092-Sevilla, Spain
}

a)luis@fa.upc.edu

Received January 24, 2019; Revised May 18, 2019; Published October 1, 2019

\begin{abstract}
Bifurcations of limit cycles in an H-bridge LC resonant inverter are reexamined taking into account a time delay in the switching transition. The analysis is accomplished by means of a model of the inverter that compresses, in only three parameters, all the elements associated to both series and parallel topologies of the inverter, to the parasitic effects, to the state feedback control, and to the switching time delay. Emphasis is made in the deviation of preexisting bifurcations without delay and the new ones arising when the time delay is taken into account. It is shown from the analysis and numerical simulations that the delay can degrade the quality of oscillations and even inhibit them, but it is also demonstrated that to some extent, this drawback can be compensated by an appropriate state feedback.
\end{abstract}

Key Words: resonant inverter, switching delay, limit cycle, bifurcations

\section{Introduction}

Switch-mode power converters are nonlinear circuits widely used for power management systems in many industrial applications such as in renewable energy technologies [1], electric and hybrid vehicles [2], efficient lighting [3] and servers telecommunications [4], among others. In particular, DC$\mathrm{AC}$ resonant converters are switched-mode power converters, in which a DC voltage is converted into an AC one by means of oscillations in an H-bridge LC resonant tank circuit. The objective of this paper is focused in this kind of circuits. Sometimes also, the AC output is converted back to a DC voltage using a diode bridge rectifier, thus resulting a DC-DC resonant converter. Due to their potential to achieve increased efficiencies and high power densities [4], resonant converters are finding applications in many emerging fields with increasing prevalence in different consumer electronics applications such as in induction heating [5], wireless power transfer technologies like in battery charging for electrical 
vehicles [2] or in implantable biomedical operations [6]. However, resonant inverters in particular, and in general switched-mode power supplies, are circuits that can exhibit complex dynamics related to the switching nonlinearity. Different causes of limit cycle instability have been documented for these circuits, for instance in $[7,8]$. Moreover, the dynamics of many power electronics systems, including the one analyzed here, can be described by a set of piecewise-linear differential equations, then belonging to the class of dynamical systems frequently named Filippov systems [9]. Even the Filippov vector field describing the dynamics at the switching manifold is smooth, it induces a nonsmooth global vector field. The propagation delay is another problem of switching converters that has rarely been taken into account. Clearly, the relevance of the propagation time delay effects increases with the switching frequency.

Many approaches used by scientists and engineers to study the dynamics of switched systems such as averaging [10], the time-domain Hamel locus [11] describing function (DF) or First Harmonic Approximation (FHA) method [12-14] fail to give accurate results whenever the repulsive sliding dynamics plays a relevant role $[15,17]$. Mostly, the sliding region, a subset of the switching manifold i.e., the region of the state-space where the field is discontinuous, is attractive and then techniques of sliding control can be applied [18]. However, in our case, the sliding region is repulsive and then, as it will be demonstrated, it plays a significant role in the boundary between the possible basins of attraction of the different coexisting steady-state solutions. To summarize, the analysis of the resonant inverter exposed in this paper can help designers to get knowledge of the regions in the parameter space for which the desired oscillatory regime is possible and the set of initial conditions in the state-space guarantying the convergence to this regime.

Concerning the time delay in a real switching, which is mainly due to capacitive effects in the physical switches and drivers, it can produce some changes in the limit cycle and even in its existence conditions. Moreover, not only bifurcations are shifted due to the presence of delay in the switching action, but also new ones emerge as it will be shown in this paper. Following our initial work in [19], a detailed analysis of the system is given in this paper. Some work related to the role of the time delay in switching systems can be found in [9]. In [20], the switching time delay is included to compute the saltation matrix and the resulting monodromy matrix in this kind of switched systems. In particular, in the power electronics field, the effect of the propagation delay in the current feedback was determined in $[8,21]$.

This paper is structured in six different sections and its outline is as follows. First, the system under study is described in Section 2 and mathematically modeled in Section 3. The equilibrium points and the dynamics of the system without taking into account the time delay effect are revisited in Section 4. Then, the analysis is extended in Section 5 by including the time delay in the switching decision. Finally, the conclusions of this study are drawn in the last section.

\section{System description and operation principle}

\subsection{Description of the circuit}

The schematic circuit diagram of the system under study is depicted in Fig. 1. The scheme can be used to encompass both series and parallel topologies. It also includes parasitic resistances in the energy storage components. The control strategy used in this paper is an extended version of the one presented at [19], where it was considered a Zero Current Switching (ZCS) strategy using only the inductor current in the control feedback. Here, the control is generalized in the sense that the output voltage also intervenes, leading to a state feedback control. In [22], the analysis of such a change in the control is provided and some advantages for the parallel topology regarding the oscillatory dynamics are examined.

The following elements can be identified in Fig. 1: the input voltage $V_{g}$; the output series resistance $R_{o s}$; the inductor with inductance $L$ and parasitic series resistor $r_{l s}$; the output parallel conductance $G_{o p}=1 / R_{o p}$; the capacitor with capacitance $C$, parasitic parallel conductance $g_{c p}=1 / r_{c p}$ and parasitic series resistance $r_{c s}$; and the switches $\mathrm{S}_{1}, \mathrm{~S}_{2}, \mathrm{~S}_{3}$ and $\mathrm{S}_{4}$. Note that for the series topology, the parallel resistor is removed $\left(G_{o p}=0\right)$ and that for the parallel topology, the series resistor is 


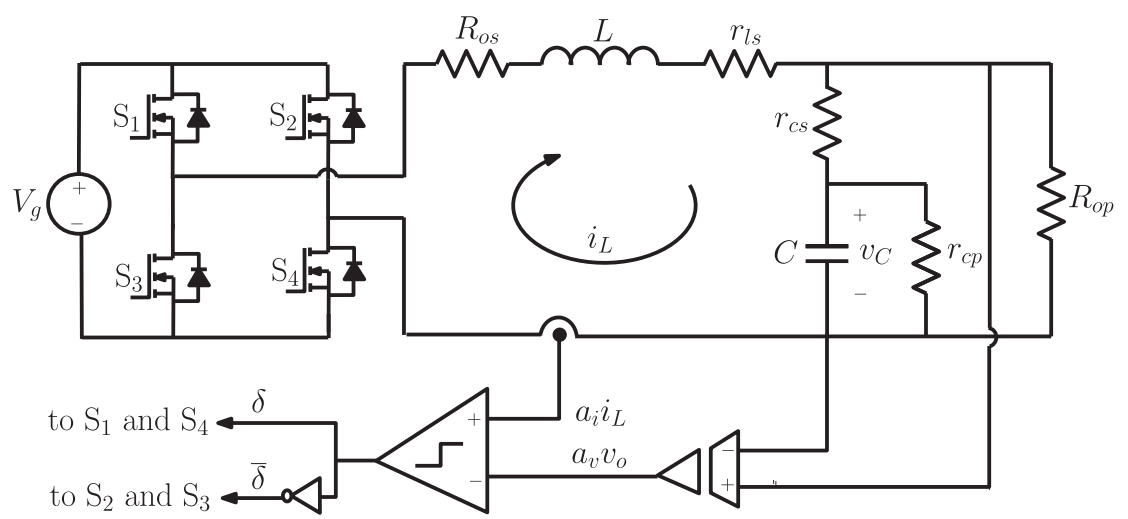

Fig. 1. Generalized schematic diagram of an H-bridge LC resonant inverter. Note that if one of the two output resistors is missing, either its corresponding conductance $\left(G_{o p}\right)$ or resistance $\left(R_{o s}\right)$ vanishes, thus leading to a series or to a parallel topology, respectively.

Table I. Parameters of the plant in the case study A with parallel configuration $\left(R_{o s}=0\right)$.

\begin{tabular}{lllllll}
\hline$V_{g}$ & $L$ & $C$ & $R_{o p}$ & $r_{l s}$ & $r_{c s}$ & $r_{o p}$ \\
$12 \mathrm{~V}$ & $1 \mu \mathrm{H}$ & $10 \mathrm{nF}$ & $33 \Omega$ & $0.1 \Omega$ & $0.1 \Omega$ & $1 \mathrm{G} \Omega$ \\
\hline
\end{tabular}
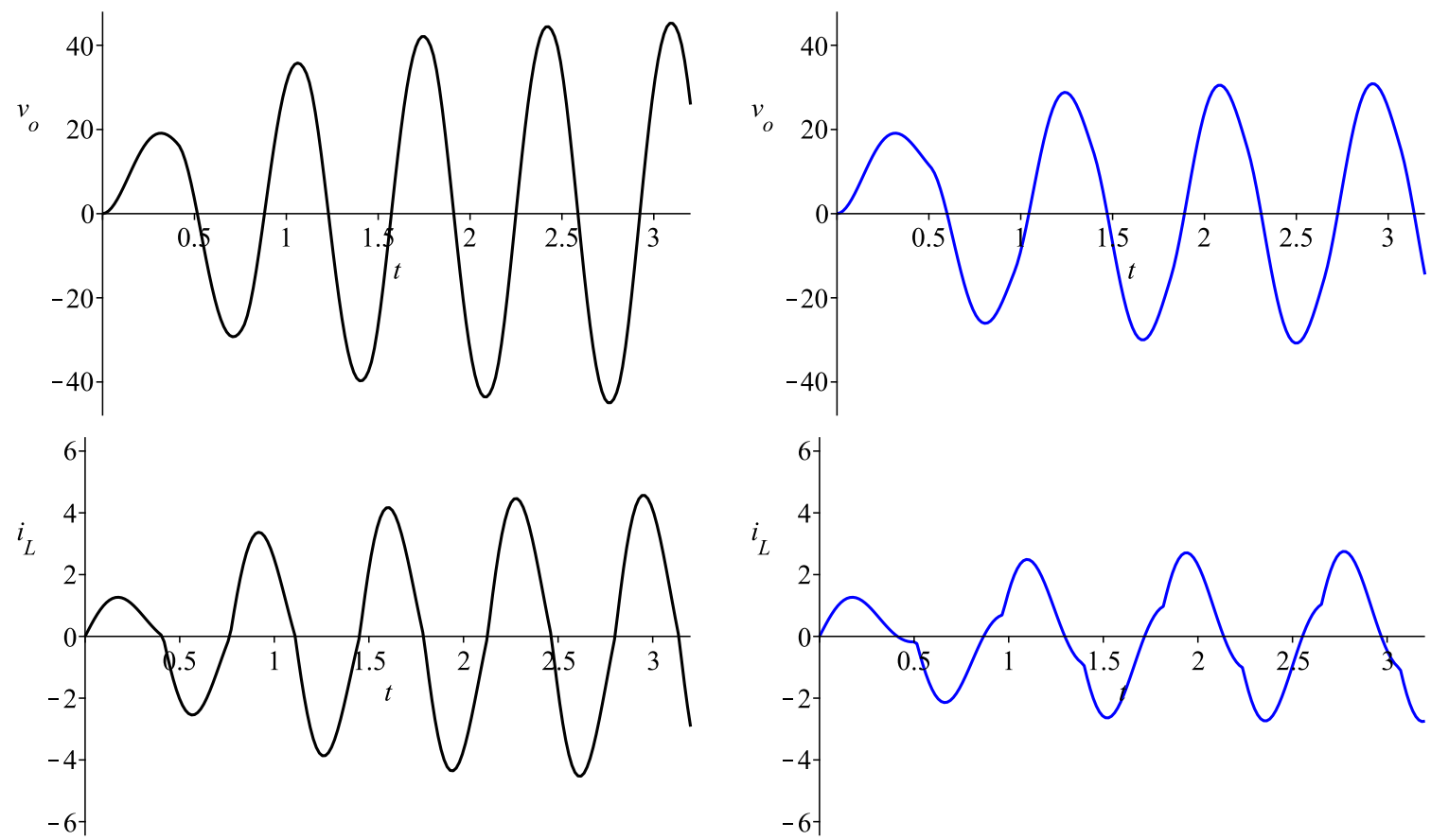

Fig. 2. Time response for circuit in Fig. 1 with parameters in Table I, $a_{v}=0$ (ZCS), no delay in left (black) panels and $0.1 \mu \mathrm{s}$ switching time delay in right (blue) panels. Units are $\mu s$ for time, $\mathrm{V}$ for the voltage output $v_{o}$, and $\mathrm{A}$ for the inductor current $i_{L}$.

excluded $\left(R_{o s}=0\right)$. Let $v_{C}$ be the voltage of the capacitor $(C), i_{L}$ be the current through the inductor $(L)$ and $v_{o}$ be the voltage in the output resistance $\left(R_{o p}\right)$. Besides, $a_{i}$ and $a_{v}$ are the gain parameters used in the state feedback control, related to the inductor current $i_{L}$ and to the output voltage $v_{o}$, respectively. Note that in this generalized model, if a pure ZCS control is used, then one has $a_{v}=0$.

The circuit operation is based on an automatically activated switching between two configurations. The switches are driven by the signal $\delta$ and its complementary $\bar{\delta}=1-\delta$. Switches $\mathrm{S}_{1}$ and $\mathrm{S}_{4}$ are ON when $a_{i} i_{L}>a_{v} v_{o}$ (and then $\delta=1$ ), and they are turned OFF when $a_{i} i_{L}<a_{v} v_{o}(\delta=0)$. Switches $\mathrm{S}_{2}$ and $S_{3}$ are driven in a complementary way with respect to $S_{1}$ and $S_{4}$. It is clear that if there is no 

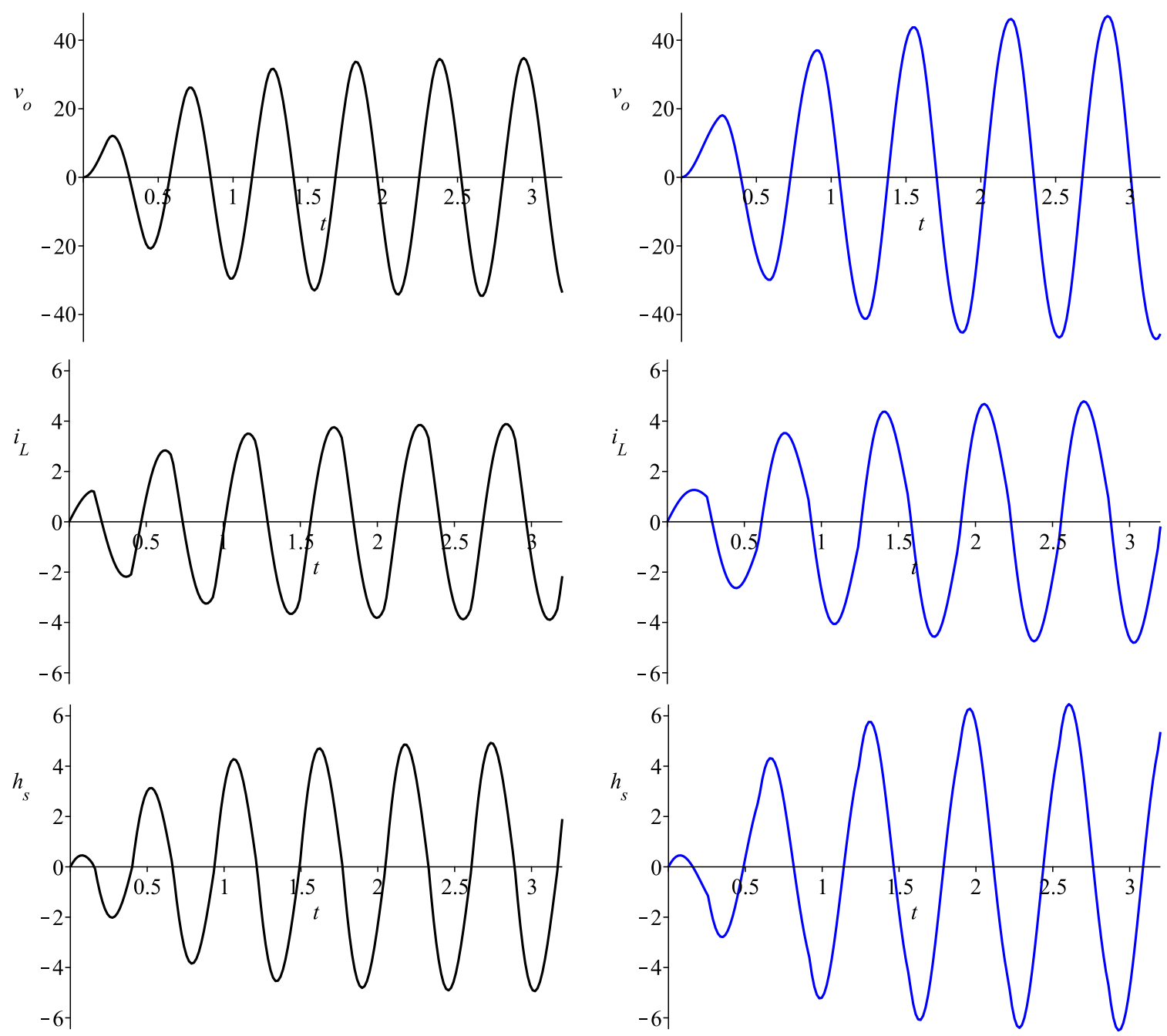

Fig. 3. Time response for circuit in Fig. 1 with parameters in Table I, $a_{v} / a_{i}=$ $0.12 \Omega^{-1}$, no delay in left (black) panels and $0.1 \mu \mathrm{s}$ switching delay in right (blue) panels. Units are $\mu s$ for time, $\mathrm{V}$ for the voltage output $v_{o}$, and $\mathrm{A}$ for the inductor current $i_{L}$ and for the control signal $h_{s}$.

switching, the capacitor voltage and the inductor current tend to their equilibrium values. Namely, there will be an equilibrium point with positive values if $\delta=1$, and its symmetrical point with negative values if $\delta=0$. Consequently, depending on the starting conditions, the dynamics could be addressed to one of these undesired equilibria. On the contrary, a limit cycle is possible when the switching is active. This oscillating regime is built by a suitable aggregation of two orbits, each one being a part of the transient regime towards the corresponding equilibrium. However, this desired objective is only achieved for certain values of the parameters and some initial conditions, as it is shown later.

\subsection{Case study A}

Let us illustrate the operation of the system by considering a first case study where the parallel configuration is assumed $\left(R_{o s}=0\right)$, being the remaining physical parameters of the circuit as specified in Table I.

The significative signals for four different situations regarding control and time delay are represented in Figs. 2-3. In Fig. 2, the control is ZCS, that is $a_{v}=0$, while in Fig. 3, it is taken $a_{v} / a_{i}=0.12 \Omega^{-1}$. For these two cases, no time delay is considered in the left (black) panels, but a switching time delay of $0.1 \mu \mathrm{s}$ exists on the right (blue) panels.

Notice that in the case with $a_{v} / a_{i}=0.12 \Omega^{-1}$ and $0.1 \mu s$ for the time delay, the amplitude and the frequency of the output are rather similar to those with ZCS and without time delay. Later on, in Section 5.1.1 this same case study will be handled in the normalized frame and further, in 
Section 5.5.1, the range of parameters will be extended to get a picture of the bifurcation pattern.

\section{Mathematical modeling}

\subsection{State-space piecewise-linear model}

First, a piecewise-linear model for the system shown in Fig. 1 will be obtained by ignoring the delay. Figure. 4 shows a simplified diagram of the system where the H-bridge output voltage is replaced by the controlled voltage source $u V_{g}$, by using the control variable $u=2 \delta-1$. Then $u=1$ when $\mathrm{S}_{1}$ and $\mathrm{S}_{4}$ are ON, otherwise $u=-1$. By applying KVL and KCL to this simplified diagram, it is straightforward that

$$
\begin{gathered}
u V_{g}=L \frac{d i_{L}}{d t}+i_{L}\left(R_{o s}+r_{l s}\right)+i_{c s} r_{c s}+v_{C}, \\
i_{L}=i_{c s}+\left(i_{c s} r_{c s}+v_{C}\right) G_{o p},
\end{gathered}
$$

where $i_{c s}$, that is the current through $r_{c s}$, is

$$
i_{c s}=C \frac{d v_{C}}{d t}+g_{c p} v_{C} .
$$

Also, the output voltage can be expressed in terms of the state variables as

$$
v_{o}=\kappa\left(v_{C}+r_{c s} i_{L}\right),
$$

in which

$$
\kappa=\frac{1}{1+r_{c s} G_{o p}} .
$$

Then, the switching condition can be expressed in terms of the state variables, $v_{C}$ and $i_{L}$, as follows

$$
a_{i} i_{L}-a_{v} v_{o}=\left(a_{i}-\kappa a_{v} r_{c s}\right) i_{L}-\kappa a_{v} v_{C}=\left(a_{i}-\kappa a_{v} r_{c s}\right)\left(i_{L}-g_{C} v_{C}\right)=0,
$$

where $g_{C}$ is an effective control factor, with conductance dimension, defined as

$$
g_{C}=\frac{a_{v} \kappa}{a_{i}-\kappa a_{v} r_{c s}}
$$

Note that the switching condition $\left(a_{i}-\kappa a_{v} r_{c s}\right)\left(i_{L}-g_{C} v_{C}\right)=0$ does not depend explicitly on time and therefore, the system is autonomous. Moreover, since $0<\kappa \leq 1$ and $r_{c s}$ is small in practice, it turns out that $\kappa$ is close to 1 and the factor $g_{C}$ differs only slightly from the ratio $a_{v} / a_{i}$. Taking also into account that $a_{i}$ must be positive in practical applications, the analysis will be restricted to the case $a_{i}-\kappa a_{v} r_{c s}>0$, so that in practice the switching manifold is determined by the ratio of the two factors, $a_{v} / a_{i}$. Then, the variable $u$ is determined by a control law such that $u=1$ (that is $\delta=1$ ) if $i_{L}>g_{C} v_{C}$, and $u=-1$ (that is, $\delta=0$ ) if $i_{L}<g_{C} v_{C}$, and so the function $h_{s}\left(i_{L}, v_{C}\right)$ associated to the switching condition can be defined as

$$
h_{s}\left(v_{C}, i_{L}\right)=i_{L}-g_{C} v_{C} .
$$

Also, by rearranging the above differential equations from KVL and KVC, the piecewise linear model becomes

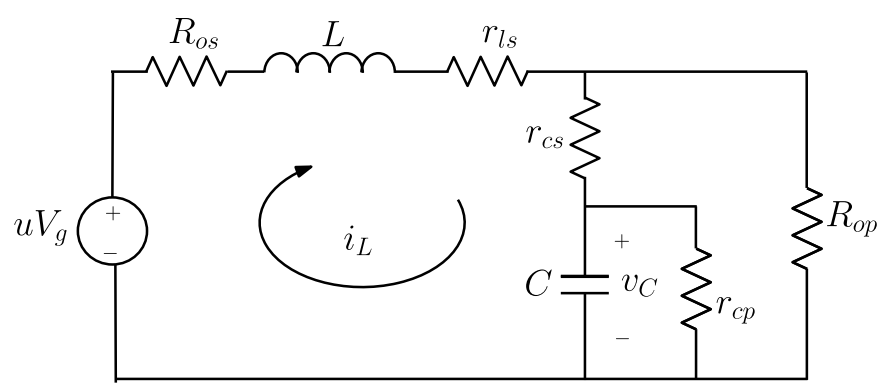

Fig. 4. Simplified diagram of the LC resonant inverter, where $u=1$ when $\mathrm{S}_{1}$ and $\mathrm{S}_{4}$ are ON, while $u=-1$ otherwise. 

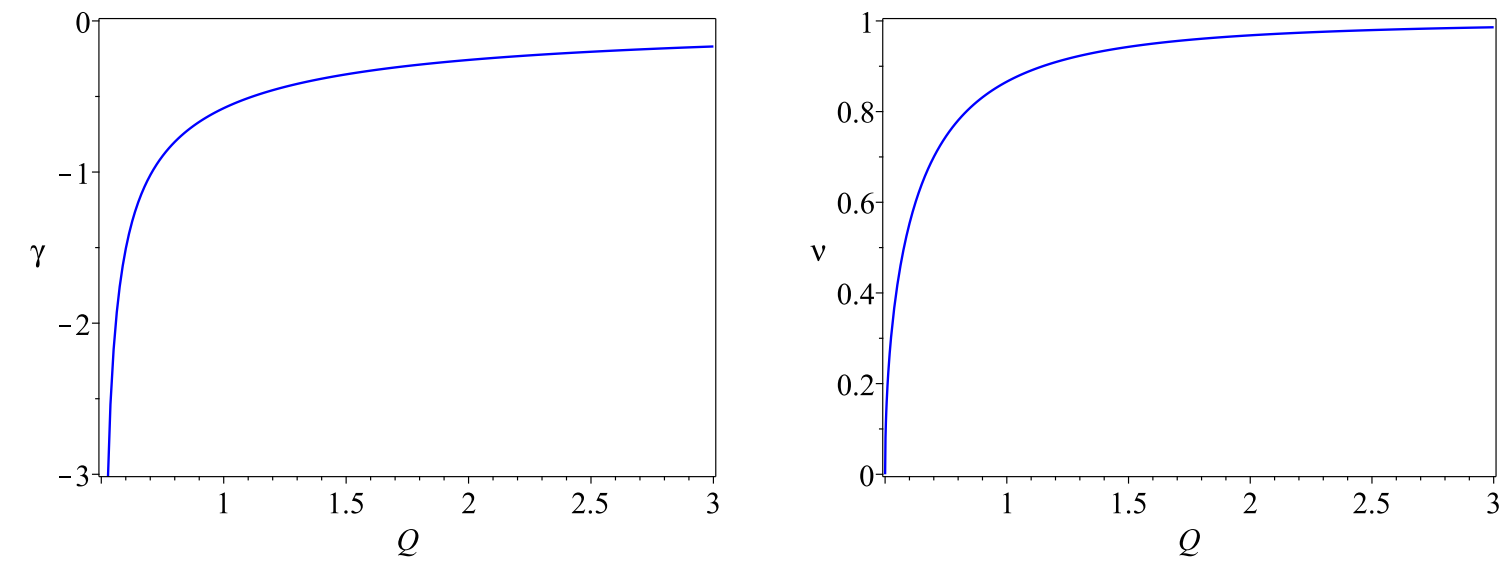

Fig. 5. The parameters $\gamma$ and $\nu$ versus $Q$.

$$
\frac{d}{d t}\left(\begin{array}{c}
v_{C} \\
i_{L}
\end{array}\right)=\mathbf{A}_{\mathbf{C}}\left(\begin{array}{c}
v_{C} \\
i_{L}
\end{array}\right)+u \mathbf{b}_{\mathbf{C}}
$$

where

$$
\mathbf{A}_{\mathbf{C}}=\left(\begin{array}{cc}
-\frac{G_{p}}{C} & \frac{\kappa}{C} \\
-\frac{\kappa}{L} & -\frac{R_{s}}{L}
\end{array}\right), \quad \mathbf{b}_{\mathbf{C}}=\left(\begin{array}{c}
0 \\
\frac{V_{g}}{L}
\end{array}\right)
$$

$R_{s}$ is the equivalent series resistance

$$
R_{s}=R_{o s}+r_{l s}+\kappa r_{c s},
$$

and $G_{p}$ is the equivalent parallel conductance

$$
G_{p}=g_{c p}+\kappa G_{o p}
$$

Let $\operatorname{det}\left(\mathbf{A}_{\mathbf{C}}\right)$ and $\operatorname{tr}\left(\mathbf{A}_{\mathbf{C}}\right)$ be the determinant and the trace of matrix $\mathbf{A}_{\mathbf{C}}$ in (3). Hence, the natural frequency $\omega_{0}$ and the quality factor $Q$ of the LC tank are

$$
\begin{gathered}
\omega_{0}=\sqrt{\operatorname{det}\left(\mathbf{A}_{\mathbf{C}}\right)}=\sqrt{\frac{R_{s} G_{p}+\kappa^{2}}{L C}}, \\
Q=-\frac{\omega_{0}}{\operatorname{tr}\left(\mathbf{A}_{\mathbf{C}}\right)}=\left(\frac{G_{p}}{\omega_{0} C}+\frac{R_{s}}{\omega_{0} L}\right)^{-1} .
\end{gathered}
$$

The case $Q>1 / 2$ is the most interesting one in applications, and so we assume such inequality in the sequel.

For convenience, the parameters $\gamma$ and $\nu$ are defined as follows

$$
\begin{gathered}
\gamma=\frac{-1}{\sqrt{4 Q^{2}-1}}, \\
\nu=\frac{-1}{2 Q \gamma}=\sqrt{1-\frac{1}{4 Q^{2}}} .
\end{gathered}
$$

Notice that $\nu \omega_{0}$ is the damped oscillation frequency for each one of the linear subsystems in (2). The two parameters $\gamma$ and $\nu$ are represented in Fig. 5 in terms of the quality factor $Q$.

Below, system (1)-(2) will be redefined in a canonical form.

\subsection{Canonical form}

In order to simplify the switching condition, let us first redefine the state variables in the form

$$
\tilde{v}=v_{C}, \quad \tilde{i}=i_{L}-g_{C} v_{C},
$$

then system (1)-(2) becomes 


$$
\begin{gathered}
\frac{d}{d t}\left(\begin{array}{c}
\tilde{v} \\
\tilde{i}
\end{array}\right)=\tilde{\mathbf{A}}\left(\begin{array}{c}
\tilde{v} \\
\tilde{i}
\end{array}\right)+u \tilde{\mathbf{b}}, \\
h(\tilde{v}, \tilde{i})=\tilde{i},
\end{gathered}
$$

where the matrix $\tilde{\mathbf{A}}$ and vector $\tilde{\mathbf{b}}$ are given by

$$
\tilde{\mathbf{A}}=\left(\begin{array}{cc}
\frac{\kappa g_{C}-G_{p}}{C} & \frac{\kappa}{C} \\
-\frac{\kappa}{L}-g_{C}\left(\frac{R_{s}}{L}+\frac{\kappa g_{C}-G_{p}}{C}\right) & -\frac{R_{s}}{L}-\frac{\kappa g_{C}}{C}
\end{array}\right), \quad \tilde{\mathbf{b}}=\mathbf{b} .
$$

Notice that the natural frequency $\omega_{0}$ and the quality factor $Q$ remain unchanged, since matrices $\mathbf{A}_{\mathbf{C}}$ and $\tilde{\mathbf{A}}$ are similar.

In searching for a canonical form as in [23], let us define the dimensionless time variable and state vector as follows

$$
\begin{gathered}
\theta=\nu \omega_{0} t, \\
\mathbf{x}=\left(\begin{array}{c}
x_{1} \\
x_{2}
\end{array}\right)=\left(\begin{array}{cc}
\frac{C\left(\kappa+g_{C} R_{s}\right)+g_{C} L\left(\kappa g_{C}-G_{p}\right)}{C V_{g}} & \frac{L\left(\kappa g_{C}-G_{p}\right)}{C V_{g}} \\
0 & \frac{\nu \omega_{0} L}{V_{g}}
\end{array}\right)\left(\begin{array}{c}
\tilde{v} \\
\tilde{i}
\end{array}\right) .
\end{gathered}
$$

Let us also define a new parameter $\beta$ as follows

$$
\beta=\frac{\left(G_{p}-\kappa g_{C}\right) L}{L G_{p}+C R_{s}},
$$

which can be varied by changing the control factor $g_{C}$. In particular, under ZCS control, $g_{C}=0$ and then $0 \leq \beta \leq 1$, with $\beta$ close to 0 for the series topology or $\beta$ close to 1 for the parallel one. As it will be shown later, the possibility of oscillation is related to the parameter $\beta$, mainly for low values of the quality factor $Q$. In this sense, by means of the state feedback control, $\beta$ can be conveniently adjusted to any real number by varying $g_{C}$. Therefore, the following result is obtained.

Proposition 1 System (8)-(9) becomes as follows

$$
\begin{gathered}
\frac{d \mathbf{x}}{d \theta}=\mathbf{A} \mathbf{x}+u \mathbf{b}, \\
h(\mathbf{x})=x_{2},
\end{gathered}
$$

where matrix $\mathbf{A}$ and vector $\mathbf{b}$ are given by

$$
\mathbf{A}=\left(\begin{array}{cc}
0 & 1+\gamma^{2} \\
-1 & 2 \gamma
\end{array}\right), \quad \mathbf{b}=\left(\begin{array}{c}
2 \beta \gamma \\
1
\end{array}\right)
$$

and $u=1$ if $h(\mathbf{x})>0$, and $u=-1$ if $h(\mathbf{x})<0$.

Note that the signal $u$ changes whenever the orbit crosses the switching manifold $\Sigma$ given by

$$
\Sigma=\left\{\mathbf{x}=\left(x_{1}, 0\right), x_{1} \in \mathbb{R}\right\},
$$

which splits the state-plane in the two regions

$$
\begin{aligned}
& \Sigma^{+}=\left\{\mathbf{x}=\left(x_{1}, x_{2}\right), x_{2}>0\right\}, \\
& \Sigma^{-}=\left\{\mathbf{x}=\left(x_{1}, x_{2}\right), x_{2}<0\right\} .
\end{aligned}
$$

The eigenvalues of the matrix $\mathbf{A}$ are as follows

$$
\lambda^{ \pm}=\gamma \pm i .
$$

Let us now reformulate the system by including the time delay in the switching action. According to the switching function given in (12), the switching decision is performed in accordance to the strategy 
already described before, i.e. $u=1$ if $x_{2}(\theta)>0$, or $u=-1$ if $x_{2}(\theta)<0$. However, if a time delay in the switching decision is taken into account, the control signal $u$ is determined by a past state of the system. Let us consider, as an approximation to the problem, a fixed switching time delay $T_{d}$ due to switches and driving circuitry. Taking into account the time delay, the switching function expression (12) is redefined as

$$
h_{\tau}(\mathbf{x}, \theta)=h(\mathbf{x}(\theta-\tau))=x_{2}(\theta-\tau),
$$

where $\tau$ is the normalized switching time delay defined as follows

$$
\tau=\nu \omega_{0} T_{d}
$$

The switching decision becomes now in such a way that $u=1$ if $x_{2}(\theta-\tau)>0$, or $u=-1$ if $x_{2}(\theta-\tau)<0$.

Then, the canonical form of the system taking into account the time delay in the switching decision can be summarized as follows

Proposition 2 System (1)-(2) with a fixed time delay $T_{d}$ in the switching action can be reduced to the piecewise linear field defined in (11), with the switching function $h_{\tau}$ as given in (16) and the matrix $\mathbf{A}$ and the vector $\mathbf{b}$ defined in (13).

Remark 1 Under Proposition 2, the parameter space of system (1)-(2) with a fixed switching time delay, have been reduced, from the starting ten physical parameters, to three independent normalized parameters: $\gamma, \beta$ and $\tau$ defined in (6), (10) and (17) respectively.

\section{Revisiting attractors and bifurcations without delay}

\subsection{Existence of equilibrium points}

First, note that system (11) with a constant value $u= \pm 1$, has the following equilibria

$$
\overline{\mathbf{x}}^{ \pm}=\left(\bar{x}_{1}, \bar{x}_{2}\right) u= \pm\left(1-\frac{4 \beta \gamma^{2}}{1+\gamma^{2}}, \frac{-2 \beta \gamma}{1+\gamma^{2}}\right),
$$

which may also be constant solutions of the switched system, whenever $\beta>0$, since $\bar{x}_{2}>0$. This is a key question because under the existence condition in the parameter space $(\beta>0)$ and certain initial conditions which will be clarified later, these equilibria can attract the orbits of the system, instead of the desired limit cycle.

\subsection{Limit cycles: existence conditions and bifurcations}

Apart from the stable equilibria $\overline{\mathbf{x}}^{ \pm}$, another steady-state behavior of system (11)-(12) can be a limit cycle. It is known that, without delay, the only possible stable oscillation is made up by two linked trajectories, see [15]. The conditions for existence of stable and unstable limit cycles are detailed in the quoted paper; for the sake of completeness, the most relevant results are reproduced below. Let us remark that unstable limit cycles play a relevant role as they are boundaries between the basin of attraction of the equilibrium points and that of the stable limit cycle.

The state vector solution $\mathbf{x}(\theta)$ for any of the two linear configurations in (11), starting at $\mathbf{x}(0)$, can be expressed as follows

$$
\mathbf{x}(\theta)=\Phi^{ \pm}(\theta, \mathbf{x}(0))=\phi(\theta)\left(\mathbf{x}(0)-\overline{\mathbf{x}}^{ \pm}\right)+\overline{\mathbf{x}}^{ \pm}
$$

where $\phi(\theta)=e^{\mathbf{A} \theta}$ is the evolution operator. Using the expression of the eigenvalues of matrix $\mathbf{A}$, given in (15), this operator is defined as

$$
\phi(\theta)=e^{\gamma \theta}\left(\begin{array}{cc}
\cos \theta-\gamma \sin \theta & \left(1+\gamma^{2}\right) \sin \theta \\
-\sin \theta & \cos \theta+\gamma \sin \theta
\end{array}\right) .
$$

Let us recall that, without delay, the system switches whenever the orbit crosses the switching manifold $\Sigma$ defined in (14). Furthermore, $\Sigma$ can be partitioned into different sections according to the field 


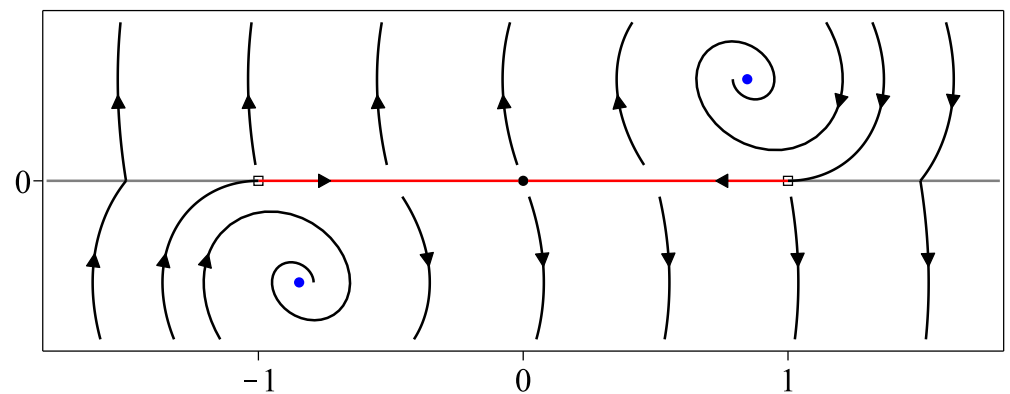

Fig. 6. Diagram of field in the vicinity of the switching manifold $\Sigma$ for system (11)-(12), with parameters $Q>1 / 2$ and $\beta>0$. Note the field points out the escaping sliding section $\Sigma^{s}$ (in red), but crosses $\Sigma$ in its crossing section $\Sigma^{c}$. The sliding field pointing from the sliding boundary (boxed points), and also the equilibrium points (small blue circles) have also been represented.

direction in its neighborhood, see Fig. 6 for an illustrative case. For the crossing section $\Sigma^{c}$, the field points to $\Sigma^{c}$ on one side and points outward $\Sigma^{c}$ on the other side. Otherwise, in the sliding section $\Sigma^{s}$, the field points either to or outward $\Sigma^{s}$ at its both sides. If the field addresses to $\Sigma^{s}$, the sliding it is said to be attractive, otherwise, it is called escaping or repulsive. In our system, the crossing and sliding partitions are found to be

$$
\begin{gathered}
\Sigma^{c}=\left\{\mathbf{x}=\left(x_{1}, 0\right),\left|x_{1}\right|>1\right\} . \\
\Sigma^{s}=\left\{\mathbf{x}=\left(x_{1}, 0\right),-1 \leq x_{1} \leq 1\right\},
\end{gathered}
$$

Moreover, the sliding subset $\Sigma^{s}$ is of escaping type, i.e., the vector field points outward at its both sides. Actually, this property justifies the relevance of $\Sigma^{s}$ in the existence of unstable sliding cycles.

Firstly, the sliding limit cycles and its associated bifurcations are described. These cycles act as boundaries between the basin of attraction of the oscillatory dynamics (stable limit cycle) and that of the equilibrium points. They are defined by an orbit partly located in $\Sigma^{+}, \Sigma^{-}$or both, and also in $\Sigma^{s}$. More precisely, they link in backward time the two closure points of the sliding region

$$
\pm \mathbf{x}_{B}=( \pm 1,0) \in \Sigma^{s},
$$

with a point $\mathbf{x}^{s c} \in \Sigma^{s}$, and also in backward time, connects $\mathbf{x}^{s c}$ again to $+\mathbf{x}_{B}$ or $-\mathbf{x}_{B}$ moving on $\Sigma^{s}$ itself.

Let $\mathbf{x}^{s c}=\left(x_{1}^{s c}, 0\right) \in \Sigma$ be a point and $\theta_{s c}$ be a time, such that the following condition holds

$$
\Phi^{+}\left(\theta_{s c}, \mathbf{x}^{s c}\right)=\phi\left(\theta_{s c}\right)\left(\mathbf{x}^{s c}-\overline{\mathbf{x}}^{+}\right)+\overline{\mathbf{x}}^{+}=\mathbf{x}_{B}
$$

and also $\Phi^{+}\left(\theta, \mathbf{x}^{s c}\right)$ lies entirely in $\Sigma^{+}$for all $0<\theta<\theta_{s c}$.

Then, the following three regular cases are possible: if $x_{1}^{s c}<-1$ so that $\mathbf{x}^{s c} \notin \Sigma^{s}$ then there are no sliding limit cycles; if $-1<x_{1}^{s c}<0$ then there exists one sliding limit cycle which defines an invariant region including the origin and the basin of attractions of the two equilibrium points; and if $0<x_{1}^{s c}<1$ then there are two sliding limit cycles, each one encircling the corresponding equilibrium point. In the last case, the origin in the state-plane is placed outside the unstable limit cycles and, consequently, an orbit starting at this point evolve to a stable limit cycle, as desired in practical applications of this circuit.

Let us assume $\gamma<0$ as a fixed parameter and a variable parameter $\beta$ in order to force a transition in the sliding limit cycle pattern between the three situations described above. Let $\epsilon$ be an enough small real number. One transition corresponds to the critical value $\beta_{c c}(\gamma)$ such that $x_{1}^{s c}=-1$. Then, a critical crossing bifurcation [24] is produced so that the unstable crossing limit cycle existing for $\beta=\beta_{c c}(\gamma)+\epsilon$ becomes a symmetric unstable sliding limit cycle for $\beta=\beta_{c c}(\gamma)-\epsilon$. The second transition occurs for the critical value $\beta_{h c}(\gamma)$ such that $x_{1}^{s c}=0$. Then, a homoclinic bifurcation of cycles is given so that the symmetric unstable sliding limit cycle existing for $\beta=\beta_{h c}(\gamma)+\epsilon$ splits in two smaller cycles, which are symmetric with respect to each other, for $0<\beta<\beta_{h c}(\gamma)$. 


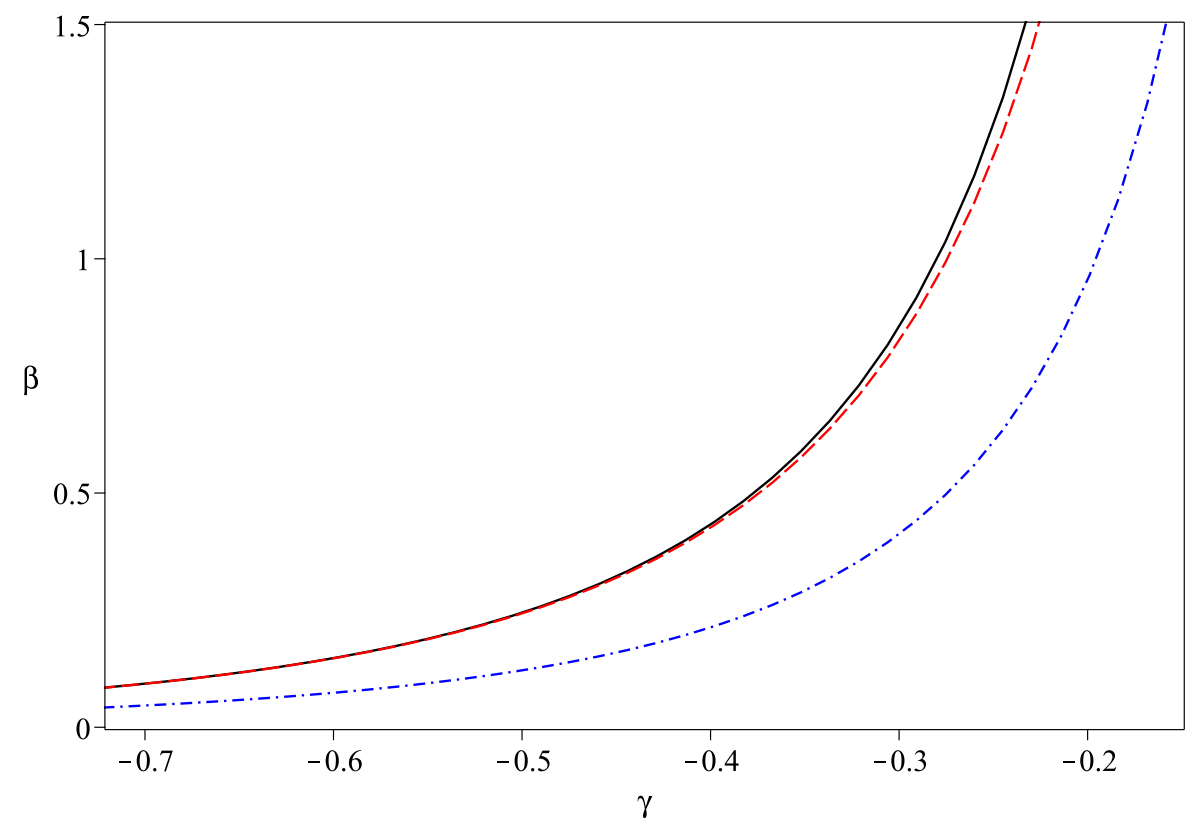

Fig. 7. Plots of the critical values of the parameter $\beta: \beta_{s n}, \beta_{c c}$ and $\beta_{h c}$ versus parameter $\gamma$, in black solid, red dashed and blue dash-dotted, which account for fold, critical crossing and homoclinic connection bifurcations respectively.

Secondly, the crossing limits cycles are addressed. These cycles, which can be either stable or unstable, can be easily obtained, taking into account the symmetry of the system, by solving

$$
\Phi^{+}\left(\theta_{c c}, \mathbf{x}^{c c}\right)=\phi\left(\theta_{c c}\right)\left(\mathbf{x}^{c c}-\overline{\mathbf{x}}^{+}\right)+\overline{\mathbf{x}}^{+}=-\mathbf{x}^{c c},
$$

where $\mathbf{x}^{c c}=\left(x_{1}^{c c}, 0\right)$ is the crossing point of the limit cycle on the left side of $\Sigma^{c}$, which evolves in $\Sigma^{+}$during a flight time $\theta_{c c}$, until reaching its symmetric point $-\mathbf{x}^{c c}$. Note that one unknown $x_{1}^{c c}$ is related to the amplitude of the oscillation and the other unknown $\theta_{c c}$ is the normalized half-period of the limit cycle.

The number of possible crossing limit cycles, as shown in [15,17], are found to be between 0 and 2 . Furthermore, if there is only one hyperbolic crossing cycle, then it is stable; if there are two crossing cycles, then the outer cycle is stable and the inner one is unstable.

By assuming also a fixed parameter $\gamma<0$, a varying parameter $\beta$ and a small real $\epsilon$, the following two bifurcations related to the crossing cycles are given. The smooth fold (also called saddle-node) bifurcation of cycles happens for the critical value $\beta_{s n}(\gamma)$ such that one non hyperbolic crossing cycle appears. Then, if $\beta>\beta_{s n}(\gamma)$ there are no oscillatory solutions, and if $\beta<\beta_{s n}(\gamma)-\epsilon$ there are two crossing limit cycles, stable the outer and unstable the inner. The second transition given for $\beta_{h c}(\gamma)$ is the homoclinic bifurcation of cycles already stated above, for which the unstable cycle changes from crossing to sliding type when simultaneously reaching the closure points of the sliding region: $+\mathbf{x}_{B}$ and $-\mathbf{x}_{B}$.

To summarize, for system (11)-(12) without time delay and from the perspective of existence and stability of limit cycles, four regions are found in the parameter plane $(\gamma, \beta)$. These regions are bounded by the three functions $\beta_{s n}(\gamma), \beta_{c c}(\gamma)$ and $\beta_{h c}(\gamma)$, which are codimension-one lines corresponding to a smooth fold bifurcation of cycles, to a critical crossing-sliding cycle and to a double homoclinic saddle connection, respectively. These three functions are depicted in Fig. 7. The complete classification of limit cycle configurations, as appearing in Theorem 1 at [15], is reproduced below, and representative pictures corresponding to each of the six cases (a)-(f) are given in Fig. 8.

(a) If $0<\beta<\beta_{h c}(\gamma)$ then there exist one stable crossing limit cycle and two unstable sliding limit cycles.

(b) If $\beta=\beta_{h c}(\gamma)$ then there exist one stable crossing limit cycle and two homoclinic connections to the origin. 


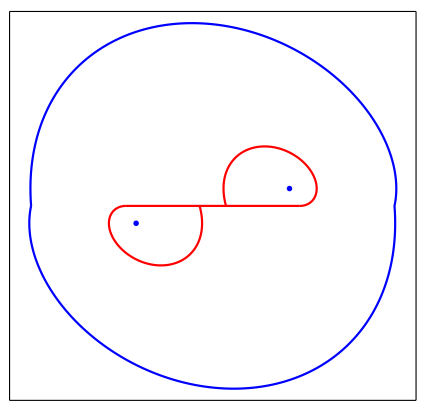

(a) $\beta<\beta_{h c}(\gamma)$

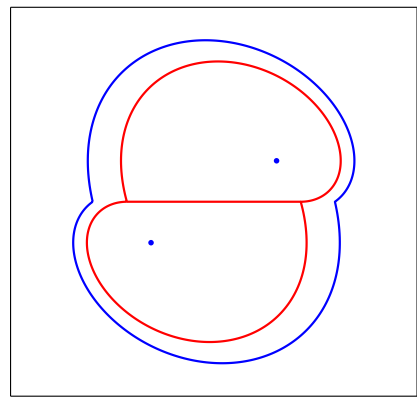

(d) $\beta=\beta_{c c}(\gamma)$

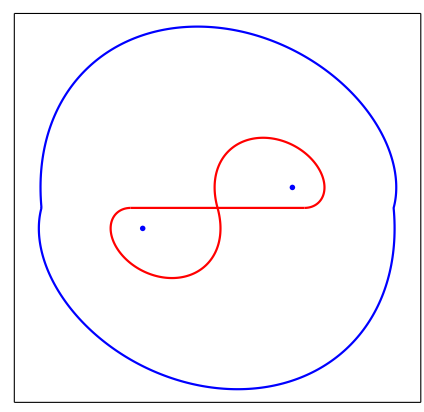

(b) $\beta=\beta_{h c}(\gamma)$

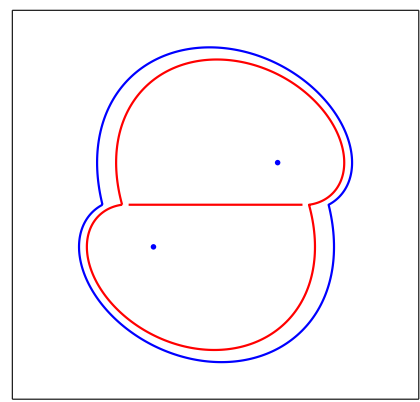

(e) $\beta_{c c}(\gamma)<\beta<\beta_{s n}(\gamma)$

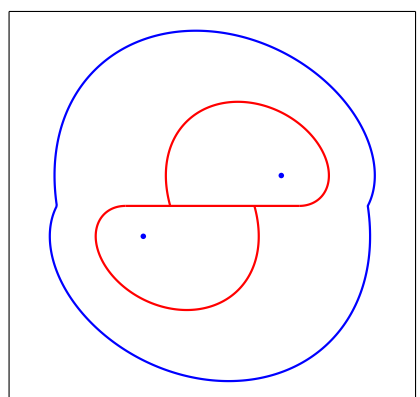

(c) $\beta_{h c}(\gamma)<\beta<\beta_{c c}(\gamma)$

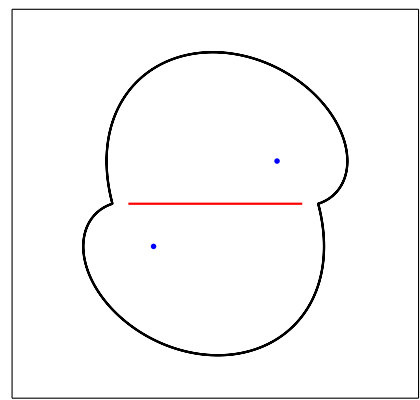

(f) $\beta=\beta_{\text {sn }}(\gamma)$

Fig. 8. Limit sets for parameters $\tau=0, \gamma$ fixed and $\beta$ given in the caption. The equilibrium points and the outer stable limit cycle are depicted in blue color, while unstable limit cycles appear in red color. The black cycle in (f) is the non hyperbolic limit cycle at the fold bifurcation. The red straight line corresponds to the sliding set $\Sigma^{s}$.

(c) If $\beta_{h c}(\gamma)<\beta<\beta_{c c}(\gamma)$ then there exist one stable crossing limit cycle and one unstable sliding limit cycle.

(d) If $\beta=\beta_{c c}(\gamma)$ then there exist one stable crossing limit cycle and one unstable critical crossing limit cycle.

(e) If $\beta_{s n}(\gamma)<\beta<\beta_{c c}(\gamma)$ then there exist two crossing limit cycles having opposite stability.

(f) If $\beta=\beta_{s n}(\gamma)$ then there is one crossing limit cycle which is semi-stable.

(g) If $\beta>\beta_{s n}(\gamma)$ then there are no crossing limit cycles.

Remark 2 The parameter condition $\beta<\beta_{h c}(\gamma)$ is the advisable one for a standard application of the inverter, because this condition implies that the origin of the state-space, which is the likely starting point for the dynamics, is not on on the basin of attraction of the equilibrium points. Moreover, to ensure that the equilibrium points are not real attractors for the switched system, the factor $g_{C}$ in the state feedback control should be chosen to get, at least during some initial time interval, a parameter value $\beta<0[16]$.

Note that for the series inverter under ZCS control, the parameter $\beta$ is a positive small value (without parasitic elements, $\beta=0$ ). Then the oscillatory dynamics is available even with reduced values of the quality factor, even if approaching critical $Q=1 / 2$.

However, for the parallel inverter under ZCS control, the value of $\beta$ is closed to 1 , then the oscillation, depending on the quality factor, could not be guaranteed. For instance, if $\beta=1$, the parameter $\gamma$ takes the critical values $\beta_{s n}^{-1}(1) \sim-0.2799, \beta_{c c}^{-1}(1) \sim-0.2744$ and $\beta_{h c}^{-1}(1) \sim-0.1954$, which correspond to the critical $Q$ values $1.8553,1.8894$ and 2.6075 , respectively. Thus to guarantee the oscillation for values of $Q$ lower than those critical, the state feedback control can be used by choosing an appropriate 
value of $g_{C}$, or equivalently of the ratio $a_{v} / a_{i}$, in order to reduce the parameter $\beta$ at least below to the critical $\beta_{h c}(\gamma(Q))$.

\section{Limit cycles with switching time delay: existence and bifurcations}

Although the switching is theoretically induced at time instants such that the orbit crosses the switching manifold $\Sigma$, actually, the transition between the two configurations is delayed due to the non-ideal features of the switches and driving circuitry, as illustrated in Fig. 9. It should be noticed that, in practice, the switching delay is relevant only for significant values of $\tau$, i.e., when $T_{d}$ is significative when compared to the period of the oscillation, like in high frequency applications.

\subsection{Some illustrative examples}

Let us first show some examples of limit cycles with different parameter sets before formalizing their direct computation and examine the possible bifurcations.

\subsubsection{Case study $\mathrm{A}$ in the normalized frame}

This first case is the same one considered in the case study A, which was introduced in Section 2.2 with physical parameters in Table I, thus resulting from (4-7), $\omega_{0} \sim 10 \mathrm{Mrad} / \mathrm{s}, Q \sim 3.1048, \gamma \sim$ $-0.1632, \nu \sim 0.9870$. Also, $\beta \sim 0.9380$ if $a_{v}=0$ or $\beta \sim-2.8101$ if $a_{v} / a_{i}=0.12 \Omega^{-1}$ and, in the switching delayed cases, from (17), $\tau \sim 0.9870$. In the following, for this case study, the constant value for the parameter $\gamma_{A}=-0.1632$ will be assumed.

Limit cycles represented in the state-space shown in Fig. 10 correspond to this case study. The topleft panel of this figure stands for limit cycles in the physical state-plane $\left(v_{C}, i_{L}\right)$ that correspond to Fig. 2, that is for $a_{v}=0$. Below, in the bottom-left panel, these orbits are depicted in the normalized state-plane $\left(x_{1}, x_{2}\right)$, thus with normalized parameter $\beta=0.9380$. En each case, black or blue orbits refer to switching time delay $T_{d}=0(\tau=0)$ or $T_{d}=0.1 \mu s(\tau=0.9870)$, respectively. The resulting limit cycles have as normalized periods $T \sim 6.6416$ if $\tau=0$ or $T \sim 8.2042$ if $\tau \sim 0.9870$.

Likewise, limit cycles in the top-right panel of Fig. 10 correspond to Fig. 3, that is for $a_{v} / a_{i}=$ $0.12 \Omega^{-1}$, and in the bottom-right panel they are represented after normalization, thus being $\beta=$ -2.8101 . Also, black and blue orbits refer to case without delay and with delay, respectively. The resulting limit cycles have as normalized periods $T \sim 5.5374$ if $\tau=0$ or $T \sim 6.4001$ if $\tau=0.9870$.

Notice that the delay can be, at least partially, compensated by the state feedback with an appropriate value of $\beta$ by adjusting the gain ratio $a_{v} / a_{i}$. The dotted straight line shown in the diagrams is

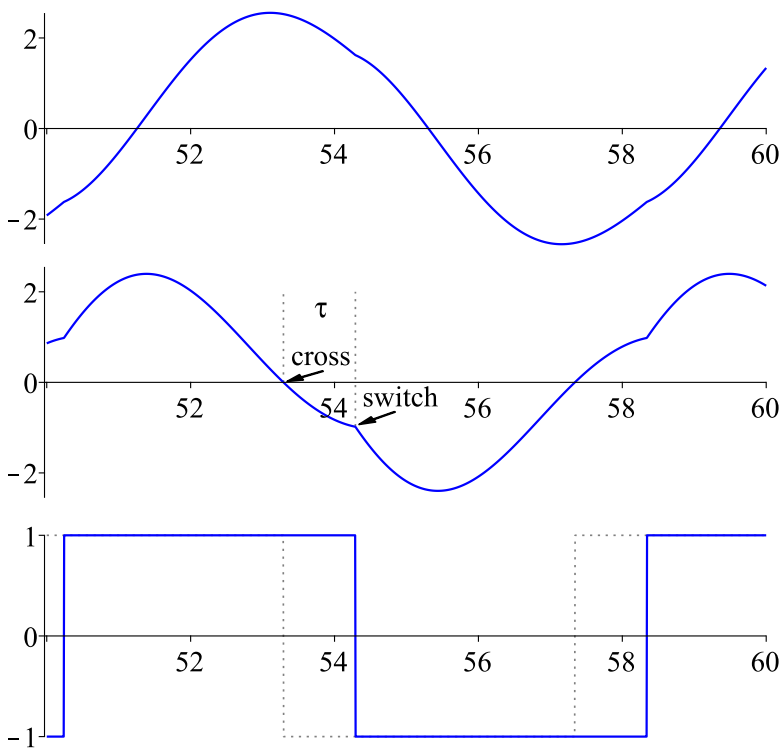

Fig. 9. Oscillograms obtained by long time simulations up to reach the steady state. Variables $x_{1}(\theta), x_{2}(\theta)$ and control $u(\theta)$ are represented from top to down. Note the time delay $\tau$ between the crossing and switching instants. 

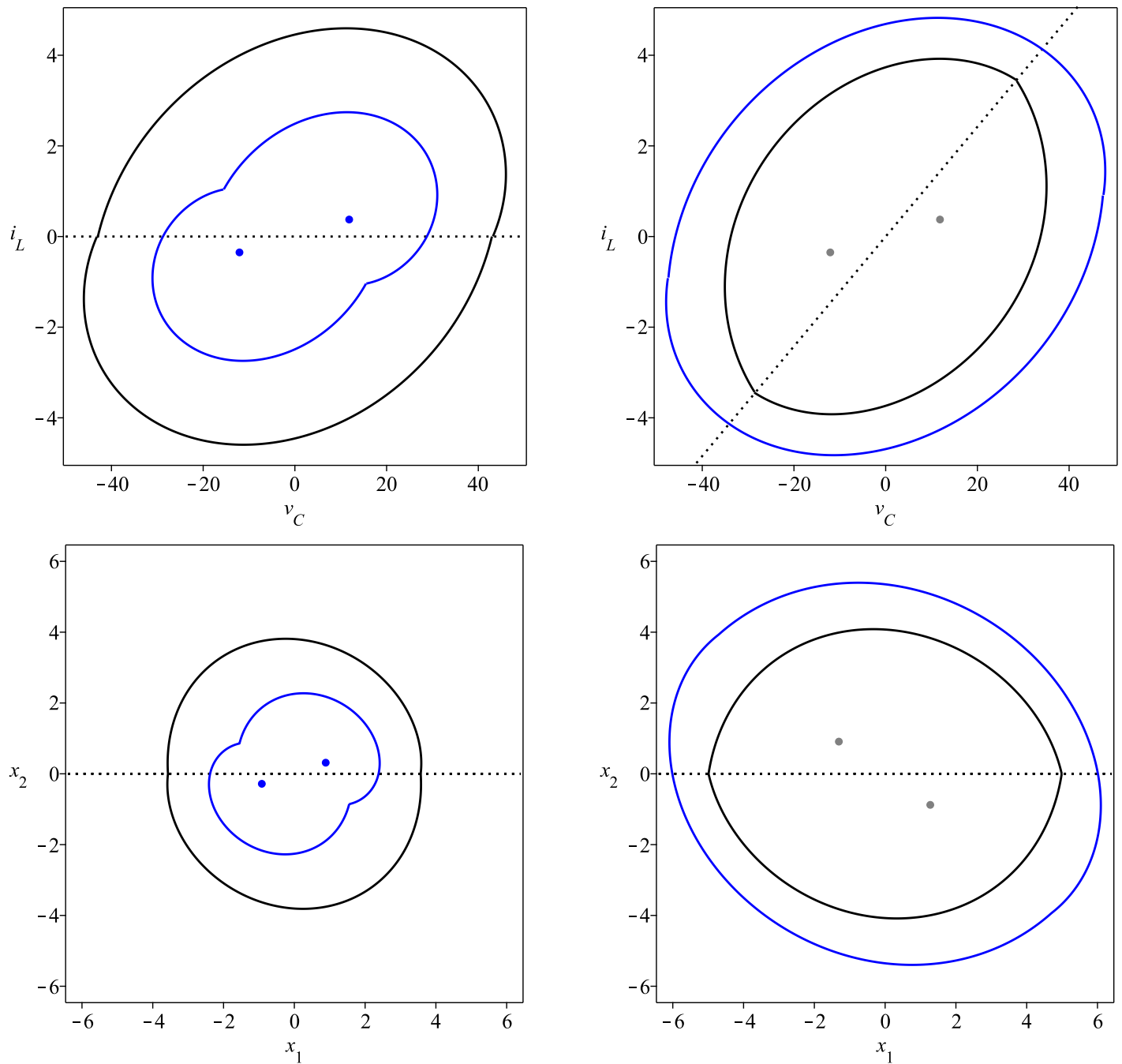

Fig. 10. Limit cycles in the state-plane with parameters in Table I. Top panels deal with physical state variables (with voltages in $\mathrm{V}$ and currents in A), and bottom ones are in normalized coordinates. Left and right panels correspond to ZCS control $\left(a_{v}=0\right)$ and to $a_{v} / a_{i}=0.12 \Omega^{-1}$, showing the steady state orbits for the waveforms represented in Figs. 2 and in 3, respectively. As in such figures, black and blue orbits stand for non delayed switching and time delay $T_{d}=0.1 \mu s$, respectively. Dots stand for equilibria, blue if real and gray if virtual; the dotted line indicates the crossing condition.

the one for crossing $(\Sigma)$, which is horizontal if $a_{v}=0$ and also, in any case in the normalized frame. The position of the equilibrium points (dots in the diagrams) regarding this line determines if they are real or virtual (marked in blue and gray colors respectively).

\subsubsection{Case study B}

This second case deals with the normalized system using a constant parameter $\beta_{B}=1$. At this stage, $\gamma=-0.15$ has also been fixed (later, in Section 5.5.2, this parameter will be varied). In Fig. 11, stable limit cycles have been computed for those fixed parameters and several values of the switching time delay $\tau$, resulting in smaller cycles as this parameter is increased. The outer cycle corresponds to the ideal case without delay, i.e., $\tau=0$ and the most internal one uses a critical value, which depends on $\beta$ and $\gamma, \tau_{c c}(\gamma, \beta) \sim 2.2526$, such that the orbit collides with $\Sigma^{s}$ just at the delayed switching instant, thus resulting in a corner-collision bifurcation [9].

If $\tau>\tau_{c c}(\gamma, \beta)$, the resonant oscillation does not exist. Instead, equilibria or other kind of non resonant oscillation (with higher frequency and lower amplitude) are possible. In the left panel of Fig. 12, the critical resonant orbit, the two equilibrium and the non resonant oscillation are shown 


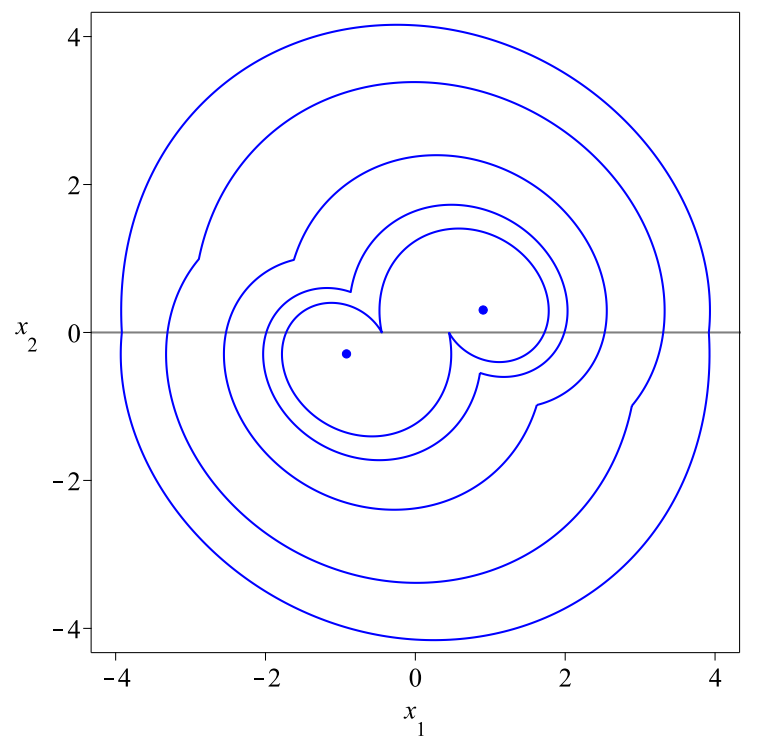

Fig. 11. (a) Plot of limit cycles with parameters $\beta=1, \gamma=-0.15$ and, from the outer cycle to the internal one, $\tau$ takes the values in the set $\left\{0,0.5,1,1.5, \tau_{c c}\right\}$. The inner two dots stand for the equilibrium points.
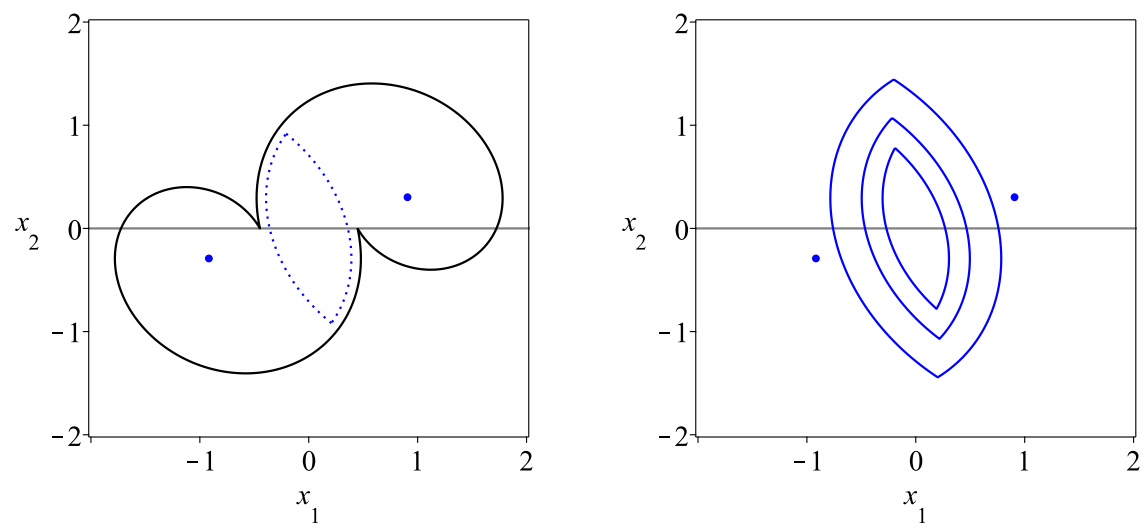

Fig. 12. Left panel stands for resonant (black continuous line) and non resonant (blue dotted line) cycles, both of them with parameter $\tau=\tau_{c c}$. Right panel does for non-resonant cycles with values of $\tau$ in the set $\{2,2.5,3\}$. In both panels, $\beta=1$ and $\gamma=-0.15$.

for the parameter set $\beta=\beta_{A}, \gamma=-0.15$ and $\tau=\tau_{c c}\left(\gamma, \beta_{A}\right)$. Also, in the right panel of Fig. 12, non resonant oscillations are plotted for several values of $\tau$ lower and higher than $\tau_{c c}$. The precise difference between these two family of limit cycles is that for those in the right panel, the switching action is delayed until the orbit crosses $\Sigma$ once more, that is $\tau>T / 2$, while in all the cases previously exposed $\tau<T / 2$, where $T$ is the normalized period of the limit cycle.

\subsection{Computation of the limit cycle with delay}

Let $\mathbf{x}^{s}=\left(x_{1}^{s}, x_{2}^{s}\right) \in \Sigma^{+}$be the point of the limit cycle, at which the switching happens a normalized time $\tau$ after the orbit has crossed the left side of $\Sigma^{c}$ at the point $-\mathbf{x}^{c}=\left(-x_{1}^{c}, 0\right)$. Taking into account the vector field symmetry, the next switching will take at the point $-\mathbf{x}^{s}=-\left(x_{1}^{s}, x_{2}^{s}\right) \in \Sigma^{-}$, a time $\tau$ after the dynamics have crossed the right side of $\Sigma^{c}$ at the point $\mathbf{x}^{c}=\left(x_{1}^{c}, 0\right)$. Consequently, if $\theta_{s}$ is the half-period of the time delayed limit cycle, it can be obtained by solving the two vectorial equations

$$
\Phi^{+}\left(\theta_{s}, \mathbf{x}^{s}\right)=-\mathbf{x}^{s}, \quad \Phi^{+}\left(\theta_{s}-\tau, \mathbf{x}^{s}\right)=\mathbf{x}^{c} .
$$

Note that the corresponding four scalar equations must be solved for the set of four unknowns $\left\{\theta_{s}, x_{1}^{s}, x_{2}^{s}, x_{1}^{c}\right\}$, therefore, with the aim to reduce the number of equations and unknowns, taking into account that $\mathbf{x}^{s}=\Phi^{-}\left(\tau,-\mathbf{x}^{c}\right)$, the limit cycle with delay under the restriction $\tau<\theta_{s}$, can be 

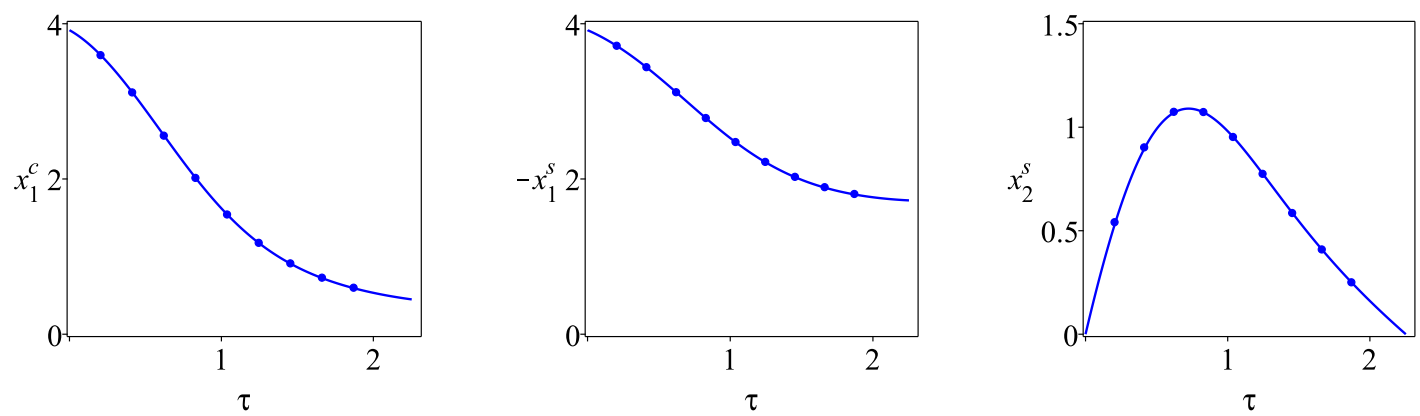

Fig. 13. Coordinates $x_{1}^{c},-x_{1}^{s}$ and $x_{2}^{s}$ illustrative of the limit cycle, versus time delay $\tau$, with parameters $\beta=1$ and $\gamma=-0.15$. Notice the agreement between direct computations using condition (22) (continuous line) and results obtained by long time dynamics simulation until reaching the steady state (dots).

obtained from

$$
\Phi^{+}\left(\theta_{s}, \Phi^{-}\left(\tau,-\mathbf{x}^{c}\right)\right)+\Phi^{-}\left(\tau,-\mathbf{x}^{c}\right)=\mathbf{0}
$$

where $\mathbf{0} \in \mathbb{R}^{2}$ is the null vector. Now the two scalar equations in (22) contains the set $\left\{\gamma, \beta, \tau, \theta_{s}, x_{1}^{c}\right\}$ of parameters and variables. In general, computations can determine two of them as a function of the other three. This permits not only to calculate the limit cycle, if it exists for a parameter set $\{\gamma, \beta, \tau\}$, but also other determinations, for instance selecting parameters to force the period of the cycle $\left(2 \theta_{s}\right)$.

In Fig. 13, $x_{1}^{c},-x_{1}^{s}$ and $x_{2}^{s}$ have been represented versus switching time delay. These curves have been computed from (22), and the dots have been obtained by long time running simulations to reasonably reach the steady state.

Concerning limit cycles for which $\theta_{s}<\tau<2 \theta_{s}$, it happens that switching due to certain $\Sigma$ crossing, from instance from $\Sigma^{-}$to $\Sigma^{+}$, is not produced while the orbit is still in $\Sigma^{+}$, but when it is in $\Sigma^{-}$ after crossing $\Sigma$ again from $\Sigma^{+}$to $\Sigma^{-}$. Then, condition (22) should be reformulated in the form

$$
\Phi^{+}\left(\theta_{s}, \Phi^{-}\left(\tau-\theta_{s},-\mathbf{x}^{c}\right)\right)+\Phi^{-}\left(\tau-\theta_{s},-\mathbf{x}^{c}\right)=\mathbf{0} .
$$

Let apply condition (23) with parameters $\gamma=-0.15, \beta=1, \tau=2.2526$, then limit cycle obtained with data $\theta_{s}=1.4862, x_{1}^{c}=0.3590, x_{1}^{s}=0.2075$ and $x_{2}^{s}=-0.9180$, is the one corresponding to the dotted line in the left panel of Fig. 12.

\subsection{Corner-collision bifurcation of the limit cycle with delay}

Let us go further with case study $\mathrm{B}$, in which $\beta=\beta_{B}=1$. Using also a fixed value $\gamma=-0.15$ in Figs. 11-13, it can be appreciated that if the parameter $\tau$ is increased, a critical resonant limit cycle collides with the switching sub-manifold $\Sigma^{s}$ (see the most internal cycle in Fig. 11). Note also, in the $x_{2}^{s}(\tau)$ representation given in the right panel of Fig. 13, that this variable becomes null for the critical parameter $\tau=\tau_{c c}(\gamma, \beta)$. When reaching this value, the resonant limit cycle collides with $\Sigma^{s}$ and after that, it is annihilated. This critical condition can be computed by (22) and the additional constraint

$$
x_{2}^{s}=\left[\Phi^{-}\left(\tau,-\mathbf{x}^{c}\right)\right]_{2}=0,
$$

thus, taking another extra parameter or variable in the set $\left\{\gamma, \beta, \tau, \theta_{s}, x_{1}^{c}\right\}$ as the third unknown. For instance, let us take the inner cycle in Fig. 11 with $\beta_{A}$ and $\gamma=-0.15$, then the corner-collision bifurcation point is found for $\tau_{c c} \sim 2.2526$, with $\theta_{s} \sim 5.9651$ and $\mathbf{x}^{c} \sim(1.7239,0) \in \Sigma^{c}$, being also $\mathbf{x}^{s} \sim(-0.4483,0) \in \Sigma^{s}$.

\subsection{Fold bifurcation of the limit cycle with delay}

Apart from the corner-collision bifurcation due to the time delayed switching, as detailed in the above section, it is also possible, in some regions of the parameter space, that a smooth fold bifurcation for limit cycles takes place. This bifurcation can be understood as an evolution of the smooth saddle-node 

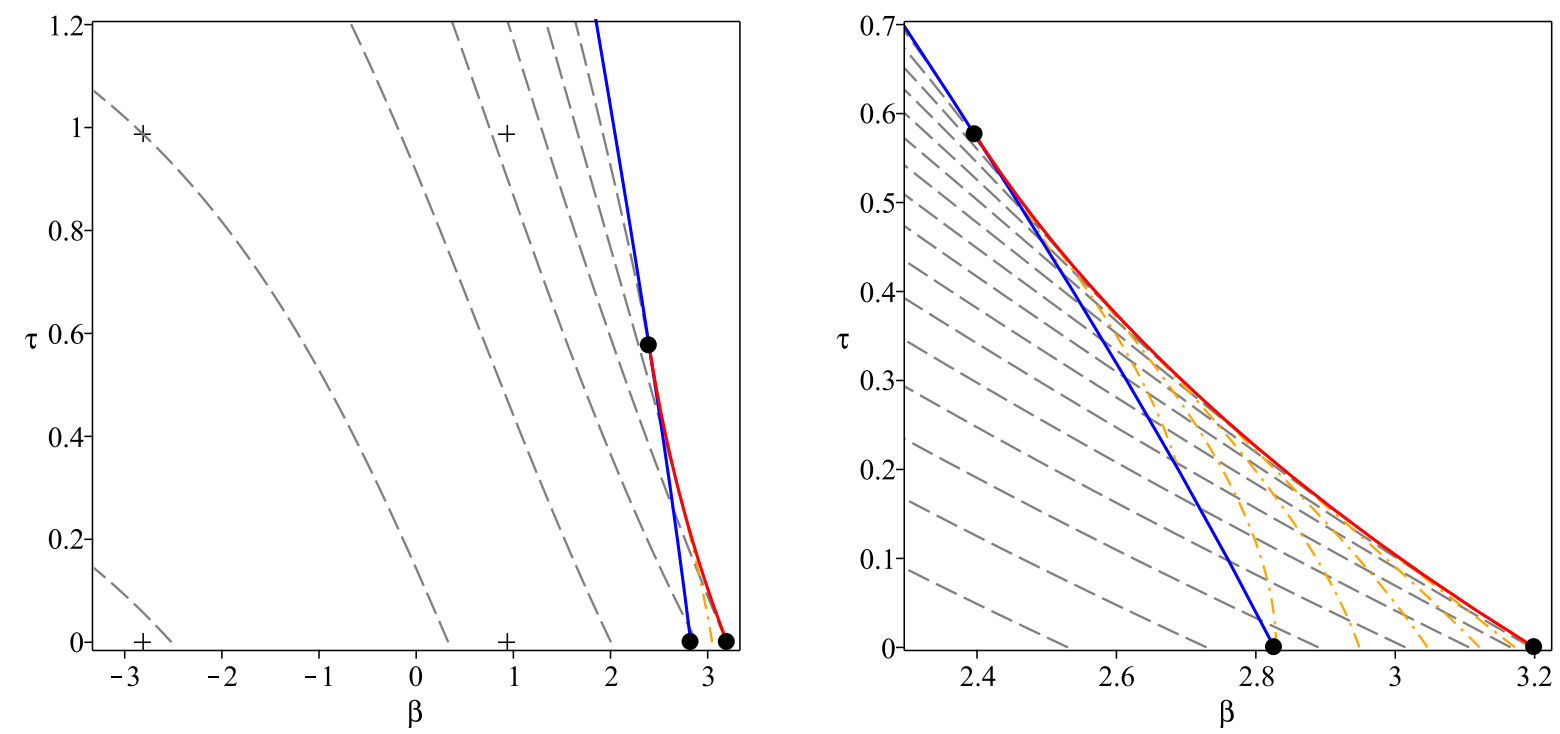

Fig. 14. Bifurcation lines (blue for corner-collision and red for saddle-node) in the parameter plane $(\beta, \tau)$ with normalized parameter $\gamma=-0.1632$ that barely approaches the value for data in Table I. Gray dashed lines give the loci of parameter points for which there exists a stable limit cycle with constant period; such lines are drawn for periods from 5.6 to 10.4 , stepped by 0.8 . The four cross-shaped points stand for the limit cycles represented in the bottom panels of Fig. 10. The diagram on the right is an enlargement to appreciate the region where stable and unstable limit cycles coexist. Here, dashed lines stand for periods from 7.6 to 10.4 stepped by 0.2 . The (dashed-dotted) orange part of these dashed lines correspond to unstable limit cycles; after being tangent to the saddle-node bifurcation line, they convert into the above mentioned gray lines for stable limit cycles.

bifurcation of cycles explained in Section 4.2. In this case, for some critical values of the parameter set $\{\gamma, \beta, \tau\}$, a non hyperbolic limit cycle exists, such that when slightly decreasing $\tau$, two crossing limit cycles, the outer stable and the inner unstable, are given. Simulations show that this bifurcation, including the switching delay, has the same qualitative features than the ideal case (without delay) given in Section 4.2 .

To get an analytical expression for this bifurcation, a zero value for $d \tau / d \theta_{s}$ can be imposed. To reach this condition, we can resort for implicit derivation rules. Let $\psi_{1}$ and $\psi_{2}$ be the two scalar functions on the left side hand in (22), then the limit cycle in the saddle-node bifurcation can be achieved from (22) itself, and in addition

$$
\frac{\partial \psi_{1}}{\partial x_{1}^{c}} \frac{\partial \psi_{2}}{\partial \theta_{s}}-\frac{\partial \psi_{1}}{\partial \theta_{s}} \frac{\partial \psi_{2}}{\partial x_{1}^{c}}=0
$$

\subsection{Illustrative examples of limit cycle bifurcations involving delay}

The two case study previously exposed are further developed here by expanding the range of some parameters. The first one with fixed parameter $\gamma_{A}=-0.1632$ and in the second case, the parameter fixed is $\beta_{B}=1$. Then bifurcations are examined by varying the other parameters.

\subsubsection{Case study A. Bifurcations}

Case study A with $\gamma_{A}=-0.1632$ comes from physical data in Table I. Some particular dynamics and limit cycles with some specific feedback and delay values were depicted in panels of Figs. 2 and 3, and also in Fig. 10 using the normalized system. A more global view for this case, introduced in Fig. 14, is attained with the help of a combined mixing of representations in the parameter plane $(\beta, \tau)$. On one hand, the blue and red continuous lines account for the corner-collision and saddlenode bifurcations respectively. Moreover, the gray dashed lines are the loci of parameter points with constant normalized period, which is selected from 5.6 to 10.4 stepped by 0.8 , and corresponding lines 

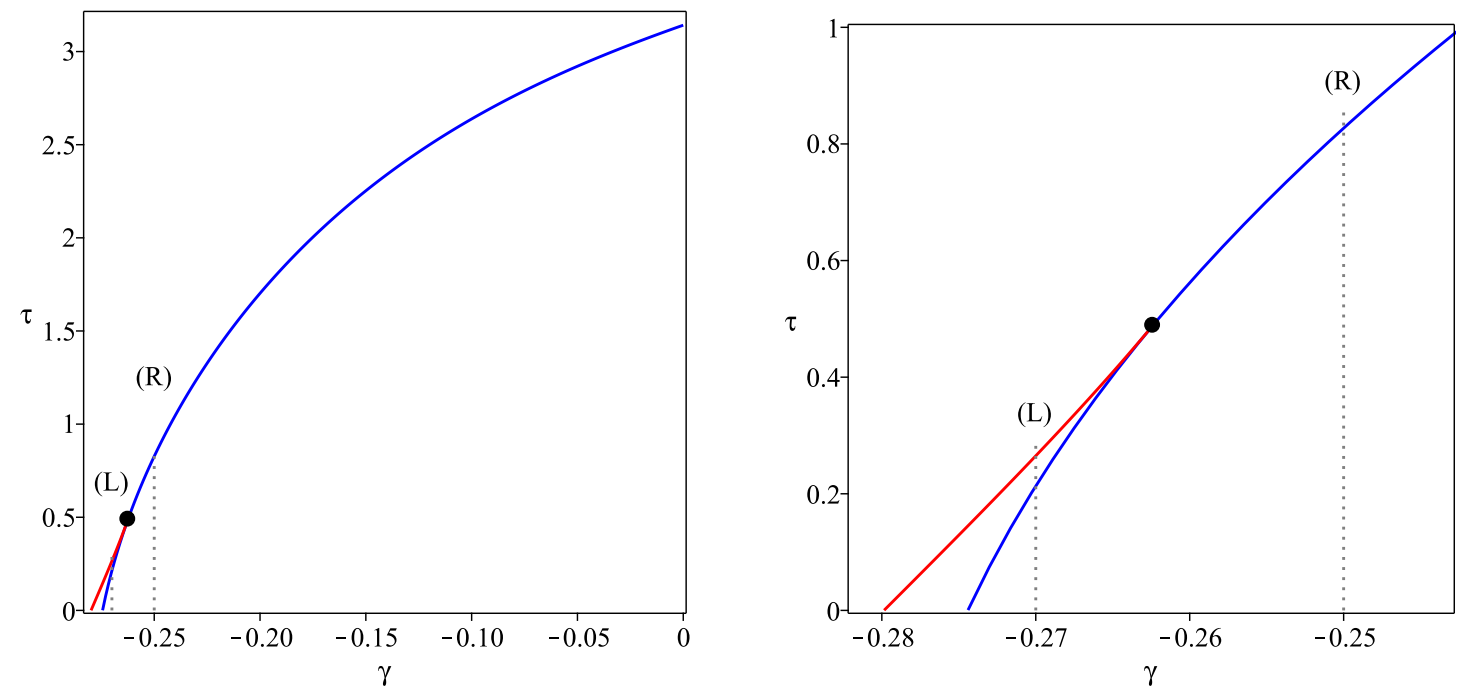

Fig. 15. Fold (red) and corner-collision (blue) codimension-one bifurcations of crossing limit cycles in the parameter plane $(\gamma, \tau)$, with fixed parameter $\beta=1$. The right panel is an enlarged view and the dot corresponds to a codimension-two bifurcation.

appear sequentially from bottom-left corner to the right side, that is the period roughly increases with delay and parameter $\beta$. All these lines start at the corner-collision bifurcation line and some of them are tangent to the saddle-node bifurcation line. Notice also the location of the four crossshaped points, which are associated to the limit cycles represented in the bottom panels of Fig. 10, for instance the one placed in the position $(\beta \sim 0.9380, \tau=0)$ corresponds to ZCS control. It can be observed that if the switching time delay increases, the shift in the period can be compensated by decreasing the parameter $\beta$ by means of the voltage feedback, as it the case of the one diagonally placed.

The right panel of Fig. 14 is just an enlargement to appreciate the region where stable and unstable limit cycles coexist. More dashed lines have been depicted representing here the set of periods from 7.6 to 10.4 stepped by 0.2 . The orange section of these lines refers to unstable limit cycles and the gray part does to stable ones, and both sides link with tangency to the saddle-node bifurcation line. Consequently, in each point inside the triangular-shaped region enclosed by the axis $\tau=0$ and the bifurcation lines, the stable and unstable part of two of these lines intersect, being the period of the unstable cycle higher than the one of the stable. Two vertices of this triangular-shaped region have as coordinates $\tau=0$ with $\beta_{c c}\left(\gamma_{A}\right) \sim 2.8264$ and $\beta_{s n}\left(\gamma_{A}\right) \sim 3.1996$. The third vertex is defined by a codimension- 2 bifurcation point belonging to the corner-collision line at which the saddle-node one tangentially ends. The parameter values for this point have been obtained from the set of four scalar equations defined in (22), (24) and (25), with resulting values $\beta_{A}^{*}\left(\gamma_{A}\right) \sim 2.3969$ and $\tau_{A}^{*}\left(\gamma_{A}\right) \sim 0.5765$.

\subsubsection{Case study B. Bifurcations}

The second case study stands for constant parameter $\beta_{B}=1$. Some results concerning this case appeared in Figs. 11 and 13. Here, a more general view is given in Figs. 15-16. In the first figure, lines for corner-collision and saddle-node bifurcations are depicted in the parameter plane $(\gamma, \tau)$. The codimension-two bifurcation point at the intersection of these lines is calculated as well, and takes the values $\gamma_{B}^{*}\left(\beta_{B}\right) \sim-0.2624$ and $\tau_{B}^{*}\left(\beta_{B}\right) \sim 0.4886$. The right panel of Fig. 15 is an enlargement for a detailed view of these lines and the intersecting point.

Let us give a complementary description so as to enhance the relevance of the codimension-2 point. To achieve this goal, two set of diagrams are represented in Fig. 16. Variables represented in these diagrams, as function of the time delay, are the half-period $\left(\theta_{s}\right)$, the crossing point $\left(x_{1}^{c}\right)$, the two coordinates of the switching point $\left(x_{1}^{s}, x_{2}^{s}\right)$ and the characteristic multiplier $(m$, which is obtained numerically by small perturbation of the limit cycle). Data in left or right panels of Fig. 16 are obtained along $\mathrm{L}$ or $\mathrm{R}$ dotted lines marked in Fig. 15. The left case is made with parameter 

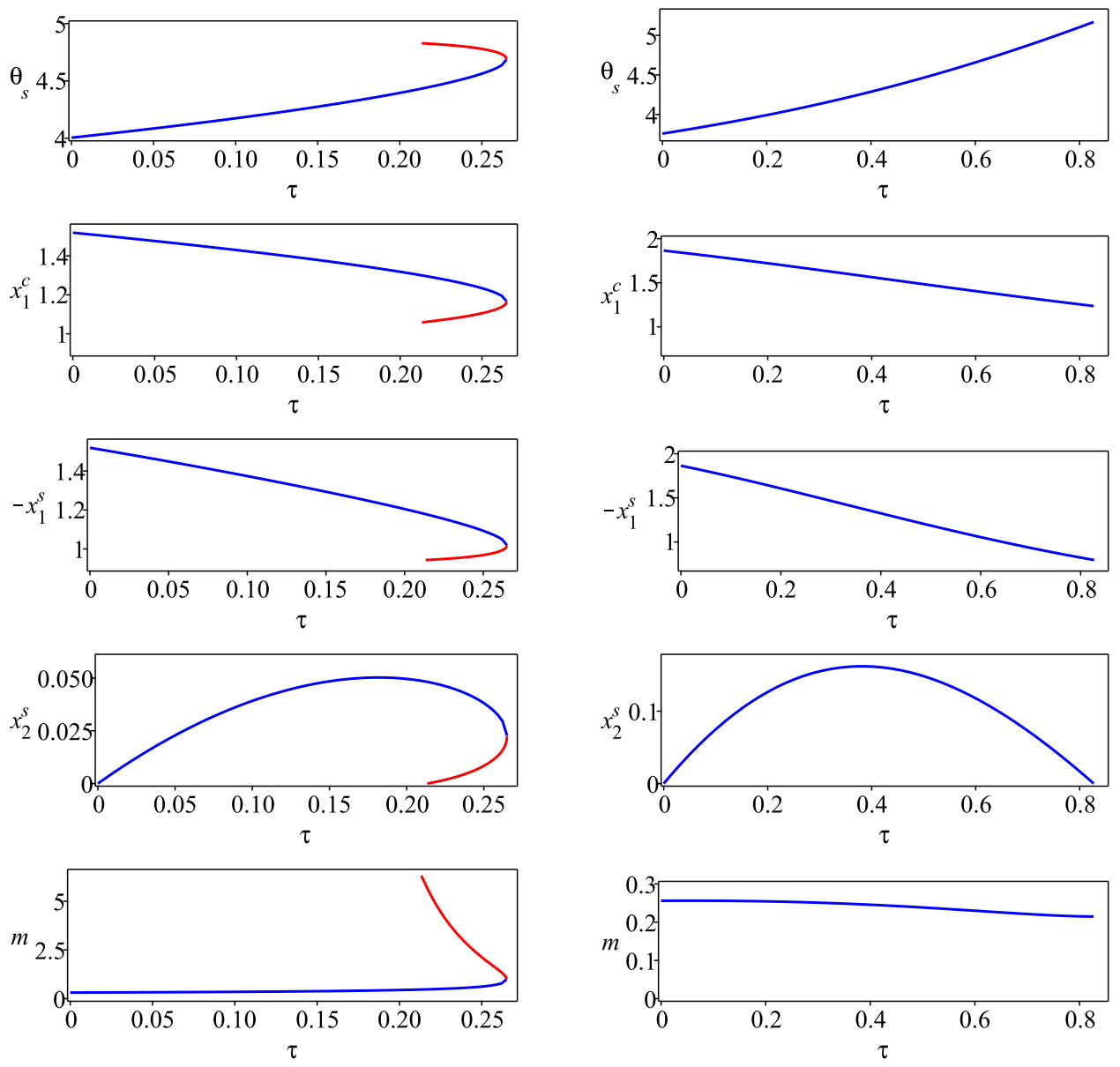

Fig. 16. Diagrams for representative variables of the limit cycles, whenever they exist, with switching time delay as a varying parameter. In both panels $\beta=1$. Besides, $\gamma=-0.27$ in the left panels and $\gamma=-0.25$ in the right panels. The existence of unstable cycles, marked in red color, only is possible for the first case. Notice that the extinction of the stable cycle (in blue color) is due to a smooth fold bifurcation in that case and to a corner-collision in the second case.

$\gamma_{L}=-0.27<\gamma_{B}^{*}$ and so the two existing limit cycles of opposite stability extinguish in a saddlenode bifurcation at $\tau_{s n}\left(\gamma_{L}, \beta_{B}\right) \sim 0.2651$. Notice also that the unstable limit cycle is bounded by a corner-collision bifurcation at $\tau_{c c}\left(\gamma_{L}, \beta_{B}\right) \sim 0.2134$. The right case is made with parameter $\gamma_{R}=-0.25>\gamma_{B}^{*}$ and so only the stable limit cycle exists and it is bounded by a corner-collision bifurcation at $\tau_{c c}\left(\gamma_{R}, \beta_{B}\right) \sim 0.8270$.

\section{Conclusions}

In this paper the limit cycle oscillation in a generic model of resonant inverters has been addressed using an exact solution for the system trajectory. First the switching case without delay has been revisited. Further, the effect of the propagation delay in the switching decision has been unveiled. It is shown that the same approach can be used for both cases, but for significant values of the switching time delay, the existence and the stability of the limit cycles can be altered. Some cases have been considered and simulated showing that the approach in this paper gives accurate results in terms of the prediction of the limit cycle behavior and its stability. In particular, a smooth fold and a cornercollision bifurcations of cycles have been predicted for an H-bridge self-resonant inverter taking into account the propagation delay in the switching decision. Some examples in terms of ad hoc suitable parameters have been computed. 


\section{Acknowledgments}

This work has been sponsored by the Spanish Agencia Estatal de Investigación (AEI) and the Fondo Europeo de Desarrollo Regional (FEDER) under grant DPI2017-84572-C2-1-R, by the Spanish Ministerio de Ciencia e Innovación under grant MTM2015-65608-P, and by the Consejería de Economía y Conocimiento de la Junta de Andalucía under grant P12-FQM-1658.

\section{References}

[1] T. Qian, "A converter combination scheme for efficiency improvement of PV systems," IEEE Transactions on Circuits and Systems - II, Express Briefs, vol. 65, no. 11, pp. 1668-1672, November 2018.

[2] L. Zhao, D.J. Thrimawithana, U.K. Madawala, A.P. Hu, and C.C. Mi, "A misalignment-tolerant series-hybrid wireless EV charging system with integrated magnetics," IEEE Transactions on Power Electronics, vol. 34, no. 2, pp. 1276-1285, February 2019.

[3] P.D. Teodosescu, M. Bojan, and R. Marschalko, "Resonant LED driver with inherent constant current and power factor correction," Electronics Letters, vol. 50, no. 15, pp. 1086-1088, July 2014.

[4] S.Y. Yu, R. Chen, and A. Viswanathan, "Survey of resonant converter topologies," 2018 Texas Instruments, Power Supply Design Seminar SEM2300, TI Literature Number SLUP376, https://www.ti.com/seclit/ml/slup376/slup376.pdf.

[5] J.M. Burdio, F. Monterde, J.R. Garcia, L.A. Barragan, and A. Martinez, "A two-output seriesresonant inverter for induction-heating cooking appliances," IEEE Transactions on Power Electronics, vol. 20, no. 4, pp. 815-822, July 2005.

[6] P. Si, A.P. Hu, S. Malpas, and D. Budgett, "A frequency control method for regulating wireless power to implantable devices," IEEE Transactions on Biomedical Circuits and Systems, vol. 2, no. 1, pp. 22-29, 2008.

[7] S. Banerjee, P. Ranjan, and C. Grebogi, "Bifurcations in two-dimensional piecewise smooth maps-theory and applications in switching circuits," IEEE Transactions on Circuits and Systems - I: Fundamental Theory and Applications, vol. 47, no. 5, pp. 633-643, May 2000.

[8] S. Banerjee, S. Parui, and A. Gupta, "Dynamical effects of missed switching in current-mode controlled DC-DC converters," IEEE Transactions on Circuits and Systems - II: Express Briefs, vol. 51, no. 12, pp. 649-654, December 2004.

[9] J. Sieber, P. Kowalczyk, S.J. Hogan, and M.di Bernardo, "Dynamics of symmetric dynamical systems with delayed switching," Journal of Vibration and Control, vol. 16, no. 7-8, pp. 11111140, June 2010.

[10] R. Erickson and D. Maksimovic, Fundamentals of Power Electronics, Lluwer, Springer, New York, 2001.

[11] D. Williams, C. Bingham, M. Foster, and D. Stone, "Hamel locus design of self-oscillating DC-DC resonant converters," IET Power Electronics, vol. 3, no. 1, pp. 86-94, January 2010.

[12] R. Bonache-Samaniego, C. Olalla, L. Martínez-Salamero, and H. Valderrama-Blavi, "Design of self-oscillating resonant converters based on a variable structure systems approach," IET Power Electronics, vol. 9, no. 1, pp. 111-119, January 2016.

[13] R. Bonache-Samaniego, C. Olalla, and L. Martínez-Salamero, "Dynamic modeling and control of self-oscillating parallel resonant converters based on a variable structure systems approach," IEEE Transactions on Power Electronics, vol. 32, no. 2, pp. 1469-1480, February 2017.

[14] H. Pinheiro, P.K. Jain, and G. Joos, "Self-sustained oscillating resonant converters operating above the resonant frequency," IEEE Transactions on Power Electronics, vol. 14, no. 5, pp. 803815, September 1999.

[15] L. Benadero, E. Ponce, A. El Aroudi, and F. Torres, "Limit cycle bifurcations in resonant LC power inverters under zero current switching strategy," Nonlinear Dynamics, vol. 91, no. 2, pp. 1145-1161, January 2018.

[16] A. El Aroudi, L. Benadero, E. Ponce, C. Olalla, F. Torres, and L. Martinez-Salamero, "Suppres- 
sion of undesired attractors in a self-oscillating H-bridge parallel resonant converters under zero current switching control," IEEE Transactions on Circuits and Systems - II: Express Briefs, vol. 66, no. 4, pp. 692-696, April 2019.

[17] A. El Aroudi, L. Benadero, E. Ponce, C. Olalla, F. Torres, and L. Martinez-Salamero, "Nonlinear dynamic modeling and analysis of self-oscillating H-Bridge parallel resonant converter under zero current switching control: Unveiling coexistence of attractors," IEEE Transactions on Circuits and Systems - I: Fundamental Theory and Applications, vol. 66, no. 4, pp. 1657-1667, April 2019.

[18] V. Utkin, J. Guldner, and J. Shijun, Sliding mode control in electromechanical systems, Taylor \& Francis, 1999.

[19] L. Benadero, F. Torres, A. El Aroudi, C. Olalla, E. Ponce, and L. Martínez-Salamero, "Delay effects on the limit cycling behavior in an H-bridge resonant inverter with zero current switching control strategy," Proc. NOLTA'18, pp. 395-398, 2018.

[20] F. Bizzarri, A. Brambilla, and G.S. Gajani, "Extension of the variational equation to ana$\log$ /digital circuits: numerical and experimental validation," International Journal of Circuits Theory and Applications, vol. 41, pp. 743-752, 2013

[21] T. Qian, "Subharmonic Analysis for Buck Converters With Constant On-Time Control and Ramp Compensation," IEEE Transactions on Industrial Electronics, vol. 60, no. 5, pp. 17801786, May 2013.

[22] L. Benadero, F. Torres, E. Ponce, and A. El Aroudi, "Dynamic analysis of self-oscillating Hbridge resonant converters with state feedback," Journal of The Franklin Institute, submitted for publication.

[23] E. Freire, E. Ponce, and F. Torres, "A general mechanism to generate three limit cycles in planar Filippov systems with two zones," Nonlinear Dynamics, vol. 78, no. 1, pp. 251-263, October 2014.

[24] E. Freire, E. Ponce, and F. Torres, "On the critical crossing cycle bifurcation in planar Filippov systems," Journal of Differential Equations, vol. 259, no. 12, pp. 7086-7107, December 2015. 